\title{
Uso do PET-CT na ginecologia: presente e futuro
}

\author{
Use of PET-CT in gynecology: present and future \\ Uso de PET-CT en ginecología: presente y futuro
}

Ana Cecília Lima Gonçalves ${ }^{1 *}$, Ana Luiza Lima Gonçalves ${ }^{1}$, Natália Oliveira Motta Salomão Alvarenga ${ }^{1}$, Manoel Monteiro de Castro Costa $^{2}$, Adirson Monteiro de Castro ${ }^{1}$.

\section{RESUMO}

Objetivo: Relatar as aplicações do PET/CT na ginecologia e sua contribuição como avanço no diagnóstico por imagem, estadiamento, reestadiamento e análise da suspeita de neoplasias. Revisão bibliográfica: Estudo de revisão sistemática descritiva, desenvolvido com produção científica indexada nas seguintes bases eletrônicas: MedLine, Up to date, Scielo, LILACS. Responde às perguntas específicas utilizando os artigos encontrados nas bases de dados citadas acima com recorte temporal de 2010 a 2019. A aplicabilidade do PET/CT na ginecologia tem sido usada e estudada para os seguintes tumores: câncer de mama, ovário, colo de útero e endométrio. Atualmente o exame é disponibilizado no SUS para neoplasia de pulmão de células não pequenas potencialmente ressecável, metástase de câncer colorretal e para o estadiamento e avaliação da resposta ao tratamento de linfomas de Hodgkin e não Hodgkin. Considerações finais: O crescimento do PET/CT dentro da área de saúde, desperta interesse e fascínio, porém seu uso exige cautela e estudos que confirmem sua eficácia, devido seu elevado custo.

Palavras-chave: Tomografia computadorizada por emissão de pósitrons, Tecnologia em saúde, Neoplasias dos genitais femininos.

\begin{abstract}
Objective: To report the applications of PET / CT in gynecology and its contribution as advances in diagnostic imaging, staging, restaging and analysis of suspected neoplasms. Bibliography review: Systematic descriptive review study, developed with scientific production indexed in the following electronic databases: MedLine, Up to date, Scielo, LILACS. Answer specific questions using the articles found in the databases cited above with a time frame from 2010 to 2019. The applicability of PET / CT in gynecology has been used and studied for the following tumors: breast cancer, ovary, cervix uterus and endometrium. Currently, the exam is available at SUS for potentially resectable non-small cell lung cancer, colorectal cancer metastasis and for the staging and evaluation of the response to the treatment of Hodgkin's and non-Hodgkin's lymphomas. Final considerations: The growth of PET / CT within the health area arouses interest and fascination, but its use requires caution and studies that confirm its effectiveness, due to its high cost.
\end{abstract}

Keywords: Positron emission computed tomography, Health technology, Female genital neoplasms.

\section{RESUMEN}

Objetivo: Reportar las aplicaciones de la PET / TC en ginecología y su contribución como avances en el diagnóstico por imagen, estadificación, reestadificación y análisis de sospechas de neoplasias. Revisión de la bibliografía: Estudio de revisión descriptiva sistemática, desarrollado con producción científica indexada en las siguientes bases de datos electrónicas: MedLine, Up to date, Scielo, LILACS. Responda preguntas específicas utilizando los artículos que se encuentran en las bases de datos citadas anteriormente con un período de tiempo de 2010 a 2019. La aplicabilidad de la PET / CT en ginecología se ha utilizado y estudiado para los siguientes tumores: cáncer de mama, ovario, cuello uterino útero y endometrio. Actualmente, el examen está disponible en el SUS para cáncer de pulmón de células no pequeñas potencialmente resecable, metástasis de cáncer colorrectal y para la estadificación y evaluación de la respuesta al tratamiento de linfomas de Hodgkin y no Hodgkin. Consideraciones finales: El crecimiento de la PET / TC dentro del área

${ }^{1}$ Faculdade de Medicina da FAMINAS BH. Belo Horizonte - MG. *E-mail: ana_cecilialima@hotmail.com

${ }^{2}$ Faculdade de Medicina Ciências Médicas de Minas Gerais. Belo Horizonte - MG.

SUBMETIDO EM: 9/2020

ACEITO EM: 10/2020

PUBLICADO EM: 10/2020

REAS/EJCH | Vol.12(10) | e5265 | DOI: https://doi.org/10.25248/reas.e5265.2020 
de la salud despierta interés y fascinación, pero su uso requiere cautela y estudios que confirmen su efectividad, debido a su alto costo.

Palabras clave: Tomografía computarizada por emisión de positrones, Tecnología de la salud, Neoplasias genitales femeninas.

\section{INTRODUÇÃO}

O exame PET/CT corresponde a uma superposição das imagens anatômicas da tomografia computadorizada (CT) aos dados da perfusão e atividade metabólica tissular proporcionados pela Tomografia por Emissão de Pósitrons (PET). É um método de diagnóstico por imagem bastante utilizado na oncologia, cardiologia e neurologia (HOCHHEGGER B e ALVES TRK, 2015).

O PET, como técnica de diagnóstico por imagem do campo da medicina nuclear, iniciou seu desenvolvimento em meados dos anos 70 . No Brasil, o primeiro equipamento de PET-CT foi instalado no Instituto do Coração do Hospital das Clínicas da Faculdade de Medicina da Universidade de São Paulo (INCOR), em 2002. Posteriormente, outros equipamentos foram adquiridos e instalados em institutos de saúde privados na cidade de São Paulo. A tecnologia continuou difundindo-se nos anos que se seguiram e foi incorporada também em outras regiões do país (VIANA, AA e SILVA HP, 2010; JÚNIOR JS e FONSECA $\mathrm{RP}, 2010)$.

Essa técnica é realizada a partir da administração de um radiofármaco, sendo o mais utilizado o Fluordesoxiglicose marcada com flúor 18 (18F-FDG), que se concentra na área a ser examinada, a qual presume-se estar com a atividade celular aumentada. Posteriormente, ocorre a emissão de raios gama a partir desta região que são detectados pelo PET-CT. O diagnóstico é obtido a partir do metabolismo glicólico celular (maior na área acometida). Com esse método, pode-se fazer estadiamento, reestadiamento, análise da suspeita de recidiva e planejamento radioterápico na oncologia (JÚNIOR JS E FONSECA RP, 2010; HOCHHEGGER B e ALVES TRK, 2015; GUARNIERI F e MARTINS HÁ, 2015).

O PET CT, como todos os exames usados na Medicina, apresenta uma série de vantagens e desvantagens. As vantagens se concentram no fato de possibilitar uma radioterapia localizada e principalmente um diagnóstico precoce. As limitações são o elevado custo e as chances de erros diagnósticos (HOCHHEGGER B e ALVES TRK, 2015; CAMARGO EE, 2005).

A incorporação do PET-CT ao SUS é recente, sendo que somente a partir de dezembro de 2014 houve incorporação do PET-CT no Sistema para alguns tipos específicos de câncer. As seguintes neoplasias estão na lista: pulmão de células não pequenas potencialmente ressecável; metástase de câncer colorretal; e para o estadiamento e avaliação da resposta ao tratamento de linfomas de Hodgkin e não Hodgkin. Essa incorporação foi mediada pela publicação da Portaria № 1.340, por parte do Ministério da Saúde. Antes disso, só era possível a realização de PET-CT no SUS, através de judicialização. Quanto ao uso na ginecologia, o exame ainda não é disponível na rede pública, mas sua utilização pelos atendimentos em rede particular tem mostrado benefícios (FERNANDES JA e LIMA AAP, 2015; CAMARGO EE, 2005).

O referente estudo objetiva revisar as principais aplicações do PET/CT na ginecologia, assim como sua contribuição no campo da medicina diagnóstica, estadiamento, reestadiamento e análise da suspeita de recidiva de neoplasias.

\section{REVISÃO BIBLIOGRÁFICA}

O exame PET-CT consiste no acoplamento das imagens da tomografia computadorizada com a avaliação da atividade metabólica detectada pelo PET. Esse processo é realizado através da marcação das substâncias com um isótopo radioativo, resultando em um radiofármaco. Existem vários radiofármacos disponíveis para exames de PET/CT, mas o mais usado na oncologia é a 18F-FDG. Essa substância é produzida por um processo complexo de radiofarmácia. Os radiofármacos são injetados no paciente e, após determinado tempo para distribuição no corpo, um aparelho (PET) é capaz de detectar a radiação liberada e evidenciar o local 
que está emitindo essa radiação no corpo (CENTRO DE IMAGEM MOLECULAR DO INCT- MEDICINA MOLECULAR, 2012; NOZAR F, 2019).

A 18F-FDG é uma molécula semelhante à glicose e após ser administrada via endovenosa, é transportada pelo sangue até as células, onde é absorvida para ser transformada em energia. Entretanto, diferentemente da glicose, a 18F-FDG entra na célula, mas é incapaz de ser transformada em energia, ficando aprisionada em seu interior. As células de maior padrão energético vão absorver mais $18 \mathrm{~F}-\mathrm{FDG}$ e, portanto, vão ser fonte radioativa detectada pelo equipamento (GUARNIERI F e MARTINS HA, 2015).

Majoritariamente, as células com caraterísticas malignas, traduzem elevado metabolismo glicolítico, quando comparadas aos tecidos hígidos. Esta discrepância no consumo de glicose permite a detecção da doença proliferativa (CENTRO DE IMAGEM MOLECULAR DO INCT- MEDICINA MOLECULAR, 2012; NOZAR F, 2019).

A utilização do PET/CT se estende para uma série de doenças. No câncer de pulmão não pequenas células o exame auxilia na avaliação de nódulos solitários e estadiamento nodal, no mesotelioma é útil no estadiamento da neoplasia. Em tumores de cabeça e pescoço e cânceres do sistema digestivo a técnica é válida para análise do tumor e definição da possível conduta cirúrgica. Já no melanoma a aplicação do PET inclui principalmente estadiamento de pacientes de alto risco (Breslow ${ }^{*}>1,5 \mathrm{~mm}$ ) e reestadiamento de pacientes com melanoma candidatos a mastectomia. O exame também é útil quando se diz respeito ao câncer da tireoide, tumores do SNC e linfomas (DRAGOSAVAC S, 2011).

Como todos os métodos diagnósticos, o uso do PET/CT conta com vantagens e desvantagens. Uma de suas principais vantagens é a possibilidade de identificação precoce de um tumor. Utilizando-se de alterações metabólicas, torna-se possível identificar inadequações, antes mesmo que sinais e sintomas sejam detectados. Essa característica do PET/CT constitui um avanço significativo para as técnicas diagnósticas da Medicina Nuclear. Além disso, esse exame, devido sua maior acurácia diagnóstica, gera grande impacto no planejamento da radioterapia, já que se identifica a área afetada com maior precisão e assim a radioterapia pode ser direcionada, evitando efeitos sistêmicos e irradiação desnecessária de estruturas adjacentes (GUARNIERI F e MARTINS HA, 2015; HOCHHEGGER B e ALVES TRK, 2015).

Outra vantagem bastante promissora relacionada ao uso do PET-CT, é a identificação de metástases, de forma mais sensível e específica. Também se observa redução no número de cirurgias desnecessárias, já que o tumor possui delimitação precisa na imagem obtida. $O$ exame se mostra mais seguro que outros (que também utilizam radiofármacos), por utilizar 18F-FDG que tem meia-vida curta, sendo isso outra característica benéfica (HOCHHEGGER B e ALVES TRK, 2015).

Quanto às limitações do PET/CT, é preciso lembrar que devido à alta taxa de captação de glicose em células normais do SNC, o exame pode ter sua especificidade rebaixada, acarretando possibilidade de erro diagnostico. Estudos já foram desenvolvidos, e propõem o uso de marcadores diferentes, como 11Cmetionina, que pode compensar essa limitação. Além disso, existem condições, como infecções e inflamações, que levam ao aumento da atividade metabólica, e assim podem levar a captação aumentada de FDG, produzindo situações desafiadoras, e aumentando a possibilidade de equívocos diagnósticos (GUARNIERI F e MARTINS HA, 2015).

Outra causa de falha no PET/CT são as armadilhas na interpretação do exame que surgem quando as lesões medem menos de duas a três vezes a resolução espacial do tomógrafo. Essas lesões aparecem menos marcadas em razão do efeito do volume parcial, igualmente a lesões que contém poucas células ou células com baixa atividade metabólica e tumores altamente diferenciados (CENTRO DE IMAGEM MOLECULAR DO INCT- MEDICINA MOLECULAR, 2012; HOCHHEGGER B e ALVES TRK, 2015).

A ampla aplicação desse método na prática diária é uma discussão constante, devido seu potencial em revolucionar a área do diagnóstico. Entretanto, seu elevado custo evidencia uma necessidade em avaliar a efetividade do exame e assim determinar se há ou não vantagens em ampliar seu uso (CENTRO DE IMAGEM MOLECULAR DO INCT- MEDICINA MOLECULAR, 2012).

Mais especificamente na área da ginecologia, o PET/CT, ainda não é amplamente usado. Todavia, mostrase promissor quando se analisa o uso em alguns casos de câncer genital, principalmente o câncer de ovário, 
devido às suas características peculiares, como por exemplo, a expressiva capacidade de gerar metástases. Em contrapartida, em alguns tipos de cânceres ginecológicos o uso do exame não é tão interessante, como ocorre no câncer de mama e no câncer de colo de útero (JÚNIOR JS e FONSECA RP, 2016; NOZAR F, 2019).

\section{Câncer de ovário}

O câncer de ovário caracteriza-se por ser o tumor ginecológico mais difícil de ser diagnosticado e o de menor perspectiva de cura, sendo, portanto, uma das afecções crônico-degenerativas mais temíveis em virtude do grau de mortalidade. É mais comum em pessoas que possuem histórico familiar da doença e em mulheres acima de 45 anos, relacionando-se mais fortemente com mutações nos genes BRCA-1 e BRCA-2. A grande maioria dos casos é diagnosticada já em estado avançado devido à ausência de sintomas na fase inicial da doença e sintomas gerais na fase avançada, como dor pélvica e constante cansaço. Devido a tais características e a grande capacidade de provocar metástases é importante discutir o uso e a contribuição do PET/CT no estadiamento desta doença (GUARNIERI F e MARTINS HA, 2015).

Em caso de recidiva o reestadiamento é fundamental para escolher o tratamento ideal, com a finalidade de melhorar a qualidade de vida e também a sobrevida da paciente com câncer de ovário. Neste aspecto, 0 PET/CT é uma técnica extremamente útil. Pesquisas sistemáticas têm sido realizadas para comprovar essa eficácia. Como exemplo tem-se um estudo realizado pela Unicamp no período de novembro de 2006 até novembro de 2010, no qual foi possível concluir que o exame de PET-CT é útil para avaliação da extensão da recidiva de carcinoma de ovário. Ele foi feito de maneira retrospectiva incluindo 45 mulheres com suspeita de recidiva de neoplasia de ovário com alterações na ultrassonografia, tomografia ou ressonância. Para confirmação da recidiva, 15 pacientes foram submetidos a cirurgia e 30 foram acompanhadas por no mínimo de 6 meses (HOCHHEGGER B e ALVES TRK, 2015).

Dentre as mulheres submetidas ao estudo, 38 resultaram em um PET/CT positivo, todas com recidiva confirmada. Três pacientes apresentavam resultados inconclusivos no exame, todos com doença. Quatro exames foram negativados, sendo que uma paciente teve recidiva confirmada e três se mantiveram sem evidências da doença. Onze mulheres com CA-125 acima do valor de referência, resultaram em US, TC ou RM normais. Todas tiveram doença confirmada, sendo que o PET/CT detectou recidiva em nove pacientes e teve resultado inconclusivo em duas. Entre as $11 \mathrm{com}$ CA-125 normal, foram evidenciadas metástase no PETCT em oito. O PET-CT detectou lesões não suspeitas em 20 das 45 mulheres submetidas ao estudo (44,4\%) (JÚNIOR JS e FONSECA RP, 2016).

O PET-CT é uma técnica bastante útil na avaliação de recidiva de neoplasia ovariana, assim como no planejamento da radioterapia ou planejamento cirúrgico. Isso é possível a partir da fusão de imagens anatômicas e metabólicas, que não é realizada nas técnicas convencionais.

\section{Câncer de mama}

O câncer de mama é a neoplasia mais incidente na população feminina, atrás somente dos tumores de pele não melanoma, sendo mais prevalente em mulheres acima de 50 anos e raro antes dos 30 anos. Entretanto tem-se observado um aumento de incidência dessa neoplasia em faixas etárias mais jovens (MIAO Y e ZHANG L, 2016; SHAMSI M e ISLAMIAN IP, 2017; RIEDL CC, 2017; ULANER GA, 2017; BITENCOURT AGF e LIMA ENP, 2014).

O diagnóstico precoce, seguido de tratamento efetivo garante um melhor prognóstico e reduz mortalidade. Todavia, na maioria dos casos os cânceres de mama são detectados tardiamente devido a negligência da população. Dessa forma, essa neoplasia maligna é bastante presente no cenário da saúde brasileira, representando cerca de 28\% de novos casos a cada ano (SHAMSI M e ISLAMIAN IP, 2017; RIEDL CC, 2017; ULANER GA, 2017; BITENCOURT AGF e LIMA ENP, 2014).

O estadiamento do câncer de mama é baseado na classificação TNM (T: tumor, N: linfonodo, M: metástase) e a abordagem de tratamento depende do estágio apresentado. A fase inicial do câncer de mama inclui pacientes com estágio clínico I, IIA, IIB. O câncer localmente avançado inclui subgrupos de pacientes com estágio clínico IIB (tumor $>5 \mathrm{~cm}$ ) e pacientes com estágios IIIA a IIIC. Presença de doença metastática simultânea identificada na apresentação inicial é classificada como estágio IV. Neste caso, os focos de 
metástase mais frequentes são: pulmão, ossos e fígado (LEITE BF e SILVEIRA DG, 2015; BITENCOURT AGF e LIMA ENP, 2014).

Tendo em vista a elevada incidência da doença em questão e o fato dessa ser na maioria dos casos diagnosticada em estágios mais avançados, possibilitando assim, maior chance de originar sítios secundários, traduz a importância do PET/CT. Dessa forma, existem estudos direcionados em avaliar a acurácia, sensibilidade e eficácia desse método diagnostico em comparação com as técnicas diagnosticas convencionais utilizadas atualmente, sendo elas, tomografia computadorizada, ultrassonografia abdominal, radiografia de tórax e cintilografia óssea (LEITE BF e SILVEIRA DG, 2015; ULANER GA, 2017; LIYANAGE $\mathrm{SH}, 2010)$.

Dentre os estudos realizados, podemos citar o Parecer Técnico-Científico (PTC) elaborado pela área de Avaliação de Tecnologias em Saúde do DECIT/SCTIE/MS. Este foi desenvolvido a partir de pesquisas em bases de dados, como, The Cochrane Library, Centre for Reviews and Dissemination (CRD) e Medline (via Pubmed), objetivando-se encontrar revisões sistemáticas (RS) e avaliações de tecnologias em saúde (ATS). Ademais, foram realizadas consulta aos membros da The International Network of Agencies for Health Technology Assessment (INAHTA) e da Rede de Evaluación de Tecnologias en Salud de las Américas (REDETSA) (LEITE BF e SILVEIRA DG, 2015).

Ficou concluído que o PET/CT não é o teste de escolha para diagnosticar lesões primarias de câncer de mama, devido sua baixa sensibilidade e especificidade em diagnosticar lesões não palpáveis ou tumores menores que $10 \mathrm{~mm}$. Entretanto é útil na detecção de metástases locais e distantes, principalmente tumores no mediastino e ossos, que são os locais mais comuns de destas lesões (MIAO Y e ZHANG L, 2016; SHAMSI M e ISLAMIAN IP, 2017; RIEDL CC, 2017).

Segundo as revisões sistemáticas, o PET-CT demonstrou sensibilidade e especificidade altas para detecção de metástase à distância, com sensibilidade superior quando comparada às técnicas convencionais. Mais especificamente, em relação à metástase óssea, que é uma das mais frequentes em portadores de câncer de mama o PET/CT também mostrou maior sensibilidade e acurácia quando comparado com a cintilografia óssea (LEITE BF e SILVEIRA DG, 2015).

Contraditoriamente, em consulta às agências membro da INAHTA e REDESTA, ficou constatado que nenhuma delas indica o PET/CT como primeira linha para detecção de metástases distantes em pacientes com câncer de mama. Entretanto, houve recomendação da sua utilização nos casos de resultados inconclusivos dos testes de imagens convencionais (LEITE BF e SILVEIRA DG, 2015).

Além de fornecer imagens diagnósticas, o aparelho também pode ser útil para avaliação da efetividade do tratamento. Segundo Gold e colaboradores, o PET/CT pode fornecer informações funcionais da resposta ao tratamento do paciente com câncer de mama considerando que as tecnologias convencionais somente demonstram as alterações morfológicas em fase tardia (LEITE BF e SILVEIRA DG, 2015).Ademais, autores recomendam realização de estudos mais aprofundados para comprovar a eficácia do PET/CT, visto que há pesquisas atuais que se mostram contraditórias e inconclusivas (LEITE BF e SILVEIRA DG, 2015).

\section{Câncer de endométrio}

O câncer de endométrio é visto como a neoplasia ginecológica mais comum nos países desenvolvidos, e é o segundo em maior grau de malignidade, atrás do câncer de colo uterino. Geralmente o prognóstico para o câncer de endométrio é favorável, visto que a maioria dos pacientes são diagnosticados no estágio inicial da doença. Os principais fatores que determinam o prognóstico são o estágio da doença, o subtipo histológico, a presença de metástase linfonodal, a profundidade da invasão miometral e a presença de envolvimento cervical (OZGU E e OZ M, 2016).

O sistema de estadiamento cirúrgico mais aceito para carcinoma endometrial pela Federação Internacional de Ginecologia e Obstetrícia (FIGO) inclui exploração abdominal, citologia peritoneal pélvica, histerectomia, salpingo-ooforectomia bilateral e linfadenectomia seletiva pélvica e aórtica, permitindo assim uma previsão adequada do prognóstico e auxiliando na determinação do tratamento ideal para cada paciente. No entanto, esses processos de estadiamento apresentam natureza invasiva. Nesse contexto, é importante a utilização de métodos menos agressivos que permitam uma avaliação minuciosa da imagem pré-operatória para 
estabelecer a terapêutica adequada, tal como o PET/CT. Esse método de diagnóstico pode ser utilizado para determinar a presença, o estágio e a malignidade do câncer de endométrio (OZGU E e OZ M, 2016).

Nos casos de carcinoma endometrial, a utilização de um método diagnóstico não-invasivo e pré-operatório que seja capaz de avaliar a extensão e disseminação da doença, é considerada de grande utilidade para determinar os candidatos para estadiamento cirúrgico e para minimizar os custos dos procedimentos. Devido às limitações dos métodos de imagem pré-operatórios convencionais, como ultrassonografia, tomografia computadorizada e ressonância magnética, a utilização do PET/CT pode ser considerada vantajosa para determinação de pacientes de baixo e alto risco, apesar de também existir limitações relacionadas a esse método, visto que a principal deficiência do PET/CT como ferramenta diagnóstica desse tipo de câncer está relacionada à baixa sensibilidade para determinação de metástase linfonodal em pacientes com câncer de endométrio (OZGU E e OZ M, 2016).

Estudos demonstram os benefícios da realização do PET/CT no câncer de endométrio baseado nos valores de SUV (valor de absorção padronizado), visto que esses valores são obtidos através dos resultados do PET/CT. O SUV é considerado um indicador de agressividade tumoral e um marcador para alterações metabólicas nos tecidos cancerígenos, ocorrendo um aumento gradual desse valor à medida que a doença evolui de estágio e grau. Esse aumento gradual pode ser uma ferramenta útil para estratificação dos pacientes (OZGU E e OZ M, 2016).

\section{Câncer de colo uterino}

O câncer do colo do útero é o terceiro tipo de câncer mais frequente entre as mulheres de 20 a 59 anos, com aproximadamente 500 mil casos novos por ano no mundo, sendo responsável pelo óbito de, aproximadamente, 230 mil mulheres por ano. Sua incidência é cerca de duas vezes maior em países menos desenvolvidos quando comparada aos países mais desenvolvidos. Esse fato justifica-se pela baixa realização de rastreamento em populações de baixo nível socioeconômico. Através do rastreamento, é possível a detecção precoce do câncer de colo uterino para que se evite ou retarde a progressão da doença, aumentando as chances de cura da paciente. Desse modo, segundo o ministério da saúde, o rastreamento desta neoplasia deve ser feito através da citologia cervical em todas as mulheres sexualmente ativas com faixa etária igual ou superior a 25 anos, medida que além de possibilitar o diagnóstico precoce, pode também prevenir a sua ocorrência através da detecção de lesões pré-neoplásicas (JÚNIOR JS e FONSECA RP, 2016; MOREIRA B, 2018).

A incidência do câncer de colo útero ainda é bastante expressiva em âmbito mundial, o que traduz elevados custos em saúde com métodos diagnósticos e tratamento. Atualmente, muito se tem discutido a respeito da utilização do $\mathrm{PET} / \mathrm{CT}$ em oncoginecologia, visto que a técnica é eficaz em diversos outros tipos de cânceres e tem revolucionado a Medicina Nuclear. Entretanto, o PET/CT é um exame muito caro, sendo necessária realização de pesquisas aprofundadas para confirmação de sua eficácia e posteriormente recomendar seu uso nesse tipo de câncer. Ademais, a utilização do PET-CT na avaliação do câncer de colo de útero não é muito encontrada na literatura sendo mais vantajoso investir na prevenção que dispensar altos gastos com um único tipo de exame que pode não ser efetivo (JÚNIOR JS e FONSECA RP, 2016; MOREIRA B, 2018).

O rastreamento é um procedimento simples, de baixo custo e alta eficácia, e reduz, por si só, o número de casos dessa neoplasia e gera menores gastos com tratamento (JÚNIOR JS e FONSECA RP, 2016; MOREIRA B, 2018).

\section{CONSIDERAÇÕES FINAIS}

O PET/CT é um exame eficaz para diagnóstico e tratamento de afecções oncoginecológicas, mas seu uso é limitado, pois, além do seu elevado custo, os métodos diagnósticos já usados em cânceres como mama e colo de útero são muito eficazes. Ademais, sua extensão ao SUS se vê limitada por onerar o sistema desnecessariamente, pois, mesmo que seja benéfico em alguns casos de câncer de ovário e endométrio, a diminuição da mortalidade pelo $\mathrm{PET} / \mathrm{CT}$, em relação aos outros métodos convencionais, ainda deve ser comprovada. 


\section{REFERÊNCIAS}

1. BITENCOURT AGF, et al. Correlation between PET/CT results and histological and immunohistochemical findings in breast carcinomas. Radiol Bras; 2014; 47 (2).

2. CAMARGO EE. Experiência inicial com PET/CT. Revista Radiologia Brasileira, 2005; 38(1).

3. 3.CENTRO DE IMAGEM MOLECULAR DO INCT- MEDICINA MOLECULAR (CIMOL). Como é o exame de PET/CT2012 Institutos Nacionais de ciência e tecnologia.

4. DRAGOSAVAC, S. PET/CT com FDG-18 em pacientes com suspeita de recidiva de carcinoma de ovário. Faculdade de Ciências Médicas da Universidade de Campinas - UNICAMP, 2011.

5. FERNANDES JA, et al. Câncer de colo uterino: Tratamento. 2011; 3 (14).

6. GUARNIERI F, MARTINS HA. Estudos sobre a utilização do exame PET/CT no estadiamento de câncer de ovário. IX EPPC - Encontro internacional de produção científica UniCesumar, 2015; 4 (8).

7. HOCHHEGGER B, et al. PET/TC em câncer de pulmão: indicações e achados. Jornal Brasileiro de Pneumologia, 2015; 41(3): 264-274.

8. JÚNIOR JS, et al. Lista de recomendações do exame PET/CT com 18F-FDG em Oncologia. Consenso entre a Sociedade Brasileira de Cancerologia e a Sociedade Brasileira de Biologia, Medicina Nuclear e Imagem Molecular. Revista Radiologia Brasileira, 2010; 43(4): 255-259.

9. KLEIN RL, et al. Ovarian cancer metastatic to the breast presenting as inflammatory breast cancer: a case report and literature review. Journal of Cancer, 2010; 1: 27-31.

10. LEITE, B. F.; et al. Uso da tomografia por emissão de pósitrons acopladas à tomografia computadorizada (PET-CT) para detecção de metástases distantes em pacientes com câncer de mama localmente avançada. Radiol BRAS 2015, 47(3).

11. LIYANAGE SH, et al. MRI and PET Scans for Primary Staging and Detection of Cervical Cancer Recurrence. Women's Health, 2010; 6 (2): 251-269.

12. MIAO Y, et al. F-FDG PET/CT fot monitoring the response os breast cancer to miR- 143- based therapeuctis by targeting tumor glycolysis. Elsevier Inc, 2016; 5 e 357.

13. MINISTÉRIO DA SAÚDE. PET-CT no Estadiamento do Câncer Pulmonar de Células Não Pequenas. CONITEC, 2013; 2 (16).

14. MOREIRA-BARROS J, et al. Radiation-Induced Uterine Carcinosarcoma after Concurrent Chemoradiotherapy for Cervical Squamous Cell Carcinoma. Revista Brasileira de Ginecologia e Obstetrícia, 2018; 40 (12): 800-802.

15. NOZAR F, et al. Análisis de la aplicación clínica de la tomografía por emisión de positrones en el ejercicio de la ginecología oncológica en el Hospital de la Mujer. Revista Médica del Uruguay, 2019; 35 (4): 4-28.

16. ÖZGÜ, E, et al. Prognostic value of 18F-FDG PET/CT for identifying high-and low-risk endometrial cancer patients. Ginekologia Polska, 2016; 87: 493-497.

17. RIEDL CC, et al. Comparison of FDG-PET/CT and contrast-enhanced CT for monitoring therapy response in patients with metastatic breast cancer. European Journal of Nuclear Medicine and Molecular Imaging, 2017 ; 44 (9): 14281437.

18. SHAMSI M, ISLAMIAN JP. Breast cancer: early diagnosis and effective treatment by drug delivery tracing. Nuclear Medicine Review, 2017; 20 (1): 45-48.

19. ULANER GA, et al. 18F-FDG-PET/CT for systemic staging of patients with newly diagnosed ER-positive and HER2positive breast cancer. European Journal of Nuclear Medicine and Molecular Imaging, 2017; 44 (9): $1420-1427$.

20. VIANA ALA, SILVA HP. Avaliando a difusão de tecnologias médicas no sistema de saúde privado no Brasil: o caso da tomografia por emissão de pósitrons (PET). Revista Brasileira de Saúde Materno Infantil, $2010 ; 10$ (1): $187-200$. 